\title{
Sum-Rate Analysis of General OFDM Downlink Channels with 1-bit Feedback per Sub-Carrier
}

\author{
Oren Somekh, Osvaldo Simeone, Alexander M. Haimovich, and Yeheskel Bar-Ness \\ Center of Wireless Communications and Signal Processing Research (CWCSPR) \\ New Jersey Institute of Technology (NJIT), Newark, NJ 07102, USA \\ Email: \{somekh, simeone, haimovich, barness\}@njit.edu
}

\begin{abstract}
The ergodic sum-rate capacity of a single-input single-output (SISO) OFDM downlink channel, with $K$ homogenous users scales as $\log \log K$ when optimal scheduling is employed under certain conditions. However, the optimal scheduling requires that the full channel state information (CSI) for all users be available to the transmitter utilizing valuable bandwidth resources. In [1], the authors show that almost the same capacity growth holds even if the feedback rate from the users to the transmitter is reduced to 1-bit per sub-carrier per fading block for a limited class of channels. In this work, we present an alternative simple analysis for a more general class of channels resulting in a closed form expression for the ergodic achievable sum-rate. The mechanism of setting suboptimal thresholds for the 1-bit quantizers is elucidated by simple lower bound to the sum-rate. Setting these sub-optimal thresholds ensures the same growth rate and non-interference limited behavior (at high-SNR regime) as that of the full-CSI scheme asymptotically with $K^{1}$
\end{abstract}

\section{INTRODUCTION}

The demand for ubiquitous high rate data communication has motivated the development of efficient wireless communication techniques over the last decade. Multi-carrier techniques, and in particular, orthogonal frequency division multiplexing (OFDM) techniques, are known for their ability to combat inter-symbol interference (ISI) resulting from the need to communicate over wideband frequency selective fading channels. Their relative simple implementation and the emergence of low-cost power efficient high performance digital signal processors, turned OFDM into the ideal baseline for wireless communication standards such as IEEE $802.11 \mathrm{a} / \mathrm{g}$ (WiFi) and the evolving IEEE 802.16 (WiMax).

A transmitter serving multi mobile terminals may use opportunistic scheduling exploiting the multi-user diversity effect to achieve higher communication rates. This idea is easily extended to multi-carrier systems where opportunistic scheduling may be done independently for each sub-carrier [2]. A difficulty in implementing opportunistic scheduling in the downlink, is the need to track the users channel state information (CSI) at the transmitter. Since the sub-channels needed to be estimated at the receivers and fed back to the transmitter, forms of limited feedback that reduce the overhead on the system resources are of interest.

Focusing on single-input single-output (SISO) setups, various methods to reduce the amount of feedback have been

\footnotetext{
${ }^{1}$ This work was supported in part by NSF grant ANI-0338788
}

suggested recently for flat and frequency selective channels [3][4][5]. Nevertheless, these partial CSI schemes are based on feedback of an $O(N)$ real numbers, where $N$ denotes the number of sub-carriers. Making these partial CSI schemes even more economical, a 1-bit feedback per sub-carrier scheme was recently proposed in [1]. According to this scheme, for each sub-carrier a threshold is used for the users to compare their sub-carriers fade power levels to. Then each user feeds back the comparison $N$ bits result to the transmitter. It is shown in [1] for channels with uncorrelated taps, that with 1-bit feedback per sub-carrier for each of the $K$ users, and carefully selected thresholds, the achievable sum-rate demonstrates the same growth rate of $\log \log K$ as the full-CSI OFDM scheme.

The analysis of the 1-bit feedback scheme presented in [1] (which is based on the analysis of a similar scheme [6] developed for single carrier SISO downlink systems communicating over flat channels) involves order statistics, resulting in an implicit expression for the ergodic sum-rate. Such an expression provides little insight into this problem. Indeed, the authors have to resort to numerical calculations to conclude the $\log K$ asymptotic behavior of the optimal thresholds. In addition, the authors apply tedious probability convergence tools to extract the asymptotic behavior of their result. Moreover, their approach is limited to the analysis of frequency selective channels which provides uncorrelated sub-channels gains in the frequency domain. This requirement leads to channels with $N$ i.i.d. taps in the time domain. Hence, long cyclic prefix should be used by the transmitter resulting in a large overhead of bandwidth and power.

The main contribution of this work is to introduce a simple analysis, which yields a closed form expression for the ergodic sum-rate of the 1-bit feedback scheme of [1]. This expression which is also valid for channels with taps that may be correlated in general, involves power allocation to sub-carriers using the long term channel statistics. Furthermore, a simple lower bound for this rate is also presented and a sub-optimal selection of the thresholds is shown to achieve asymptotically optimal performance and non-interference limited behavior when the number of users is large. Throughout this work, a homogenous model is assumed where all users share the same fading paths delays. This assumption, which is justified for setups where the main scatterers are located near the transmitting antenna, can somewhat be relaxed, and non-homogenous channels where users may experience different fading paths 
delays, are considered with some practical limitations.

The rest of the paper is organized as follows. In Section II the system model and the homogeneous channel model are presented. The 1-bit feedback per sub-carrier per fading block scheduling is described in Section III. Section IV summarizes the results of the full-CSI scheme. An expression for the ergodic sum-rate of the 1-bit feedback scheme is derived in Section B while an asymptotic analysis of this rate for large number of users and high-SNR regime is presented in Section VI. Numerical results are summarized in Section VII. The results are extended to include a non-homogenous model in Section VIII. Finally, a short discussion and some concluding remarks are elaborated in Section IX. Various derivations and proofs are included in the Appendices.

\section{SySTEM MODEL}

The downlink channel of a single isolated cell setup in which single-antenna base station transmitter is communicating with a $K$ single-antenna mobile users, is considered. We adhere to a homogeneous model where all the channels between the transmitter and the different users have identical path delays and path gain variances, which are assumed to be constant and known to both the transmitter and receivers. The channels are assumed to be i.i.d. among users, and their impulse responses are approximately constant during the coherence time of the channels which is assumed to be much longer than the respective delay spreads. The impulse response coefficients of each channel are zero-mean complex Gaussian random variables which may be correlated in general and form an ergodic process in the time index. The reader is referred to Appendix A for a detailed analysis of the channel statistics. Lastly, the zero-mean complex Gaussian additive noise processes of the channels are assumed to be i.i.d. among different users and "white" across the relevant frequency band. To combat the frequency selective channels, OFDM modulation with $N$ sub-carriers is used. The system is properly designed (with adequate cyclic prefix) in a way that inter-carrier interference (ICI) and ISI are avoided.

Accounting for the underlying assumptions, and following proper cyclic prefix removal at the receiver and discrete Fourier transform (DFT) operation, the received vector of the $k$ th user is given for an arbitrary OFDM symbol, by

$$
\boldsymbol{y}_{k}=\boldsymbol{H}_{k} \boldsymbol{x}+\boldsymbol{z}_{k},
$$

where $\boldsymbol{x} \sim \mathcal{C N}(\mathbf{0}, \boldsymbol{P})$ is the $N \times 1$ zero-mean complex Gaussian vector of the information symbols, with covariance matrix $\boldsymbol{P}$ and overall power constraint $\operatorname{tr}\{\boldsymbol{P}\} \leq N P$. The channel frequency response is the $N \times N$ diagonal matrix denoted by $\boldsymbol{H}_{k}$, with zero-mean, complex Gaussian diagonal entries $H_{k, n} \triangleq\left[\boldsymbol{H}_{k}\right]_{n, n} \sim \mathcal{C N}\left(0, \sigma_{k, n}^{2}\right)$, correlated for each user, in general. Due to the homogenous assumption, the long term statistics of each sub-carrier is identical for all users and $\sigma_{k, n}^{2} \equiv \sigma_{n}^{2}$. Furthermore, $\left|H_{k, n}\right|^{2}$ is a chi-square random variable with two degrees of freedom and expected value of $\sigma_{n}^{2}$. The frequency domain additive noise $N \times 1$ vector is denoted by $\boldsymbol{z}_{k}$, with $\boldsymbol{z}_{k} \sim \mathcal{C N}\left(\mathbf{0}, \boldsymbol{I}_{N}\right)$. The users are fully aware of only their own CSI and use a delayless reliable uplink channel to feed back their partial CSI to the base station transmitter.

\section{Scheduling Scheme}

According to the 1-bit feedback per sub-carrier scheme:

1) For each fading block (that could span over several OFDM symbols), each user feeds back $N$ bits, where the $n$th feedback bit of the $k$ th user, $b_{k, n}$, indicates if its corresponding $n$th sub-carrier fade power level has crossed a certain level $\alpha_{n}$.

2) After receiving the $N K$ feedback bits from the users, the transmitter randomly picks one user $\tilde{k}(n)$ for transmission with power $p_{n} \triangleq[\boldsymbol{P}]_{n, n}$ over the $n$th sub-carrier, from the set of users $\mathcal{S}_{n}=\left\{k: b_{k, n}=1\right\}$. In the case where $\mathcal{S}_{n}=\emptyset$, no power is allocated for this sub-carrier during the current fading block ${ }^{2}$.

3) Finally, the selected users indices vector $\{\tilde{k}(n)\}_{n=0}^{N-1}$ is sent to the users prior to the data transmission.

The threshold vector $\left\{\alpha_{n}\right\}_{n=0}^{N-1}$ and the power vector $\left\{p_{n}\right\}_{n=0}^{N-1}$ are predetermined according to the long term channel statistics $\left\{\sigma_{n}^{2}\right\}_{n=0}^{N-1}$, where the latter is selected to satisfy the overall power constraint. Finally it is noted that since during each fading block, only one user is active for each sub-carrier, a single user encoding-decoding scheme is employed across many fading blocks (and many OFDM symbols).

\section{Preliminaries}

According to a similar scheme, but with full-CSI available to the transmitter, for each fading block, all users feed back their received power level for each sub-carrier. It is well known, that the capacity achieving scheduling of the full-CSI setup is for the transmitter to pick, for each sub-carrier, the user with "best" channel for transmission with power $p_{n}$ (the amount of power is selected to meet the overall power constraint) using a complex Gaussian codebook [2]. Next, the selected user indices vector, $\{\tilde{k}(n)\}_{n=0}^{N-1}$, and the power allocation vector, $\boldsymbol{p}=\left\{p_{n}\right\}_{n=0}^{N-1}$, are fed back to the users prior to the data transmission. Using the observation that the maximum of $K \gg 1$ i.i.d. chi-square random variables with two degrees of freedom is approximated by $\log K$ with high probability [7], it is shown in [8], that for $K \gg 1$, the ergodic sum-rate capacity per sub-carrier of the full-CSI OFDM system is given by

$$
C_{\text {fcsi }} \underset{K \gg 1}{\cong} \max _{|\boldsymbol{p}| \leq N P} \frac{1}{N} \sum_{n=0}^{N-1} \log \left(1+p_{n} \sigma_{n}^{2} \log K\right) .
$$

The solution to this standard optimization problem is given by waterfilling

$$
p_{n}=\left(\frac{1}{\lambda}-\frac{1}{\sigma_{n}^{2} \log K}\right)^{+} \text {s.t. }|\boldsymbol{p}|=\sum_{n=0}^{N-1} p_{n}=N P
$$

${ }^{2}$ Later on it is argued that by proper selection of the thresholds, the probability of a sub-carrier to be inactive vanishes when $K$ is large. Hence, the potential improvement on performance taking into account the additional available power, is negligible. 
where $(x)^{+} \triangleq \max (0, x), \quad x \in \mathbb{R}$. Examining (3) and assuming large number $N$ of sub-carriers, and $o(N)$ number of sub-carriers with long term zero fade power level $\sigma_{n}^{2}=0$, reveals that for a finite $P$ and increasing number of users $K$, the waterfilling solution converges to a uniform power allocation $p_{n}=P, \forall n$

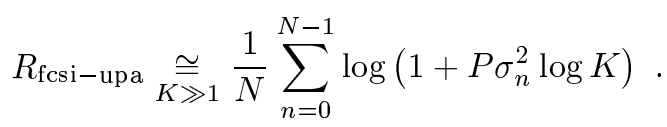

Now, since

$$
\frac{R_{\mathrm{fcsi}-\mathrm{upa}}}{\log \log K} \underset{K \rightarrow \infty}{\rightarrow} 1
$$

it is concluded that the ergodic sum-rate capacity of the full-CSI scheme grows as $\log \log K$ asymptotically with the number of users.

Examining (3) and adhering to similar argumentation regarding the channel statistics, it is concluded that waterfilling has little effect at the high-SNR regime, and uniform power allocation is optimal. In this case the high-SNR ergodic sumrate capacity slope [9] of the optimal full-CSI scheme is given by

$$
S_{\infty}^{\mathrm{fcsi}}=\lim _{P \rightarrow \infty} \frac{R_{\mathrm{fcsi}-\mathrm{upa}}}{\log P}=1 .
$$

This result holds for any $K$, not necessarily large [8].

\section{Achievable Sum-Rate}

Under the assumptions of Sections II, The ergodic achievable sum-rate per sub-carrier of the 1-bit feedback scheme described in Section III, is given by (see Appendix B for details)

$R_{1 \mathrm{bit}}(\boldsymbol{\alpha})=\max _{\boldsymbol{p}:|\boldsymbol{p}| \leq N P} \frac{1}{N} \sum_{n=0}^{N-1} Q\left(\alpha_{n} / \sigma_{n}^{2}, K\right) \mathcal{F}\left(\alpha_{n} / \sigma_{n}^{2}, \sigma_{n}^{2} p_{n}\right)$

where

$$
\begin{aligned}
& Q(x, y) \triangleq 1-\left(1-e^{-x}\right)^{y} \\
& \mathcal{F}(x, y) \triangleq \log (1+x y)+e^{\left(\frac{1+x y}{y}\right)} \operatorname{Ei}\left(\frac{1+x y}{y}\right),
\end{aligned}
$$

and $\operatorname{Ei}(x)=\int_{x}^{\infty} \frac{e^{-t}}{t} d t$ is the exponential integral function. The exact expression for the achievable rate, is valid for any $K$ (not necessarily large) and any choice of the thresholds $\boldsymbol{\alpha}=$ $\left\{\alpha_{n}\right\}_{n=0}^{N-1}$. The optimal threshold vector $\alpha$ and optimal power allocation vector $\boldsymbol{p}=\left\{p_{n}\right\}_{n=0}^{N-1}$ that maximize the achievable sum-rate $R_{1 \text { bit }}(\boldsymbol{\alpha})$, are functions of the number of users $K$, the total power constraint $N P$, and the long term channel statistics $\left\{\sigma_{n}^{2}\right\}_{n=0}^{N-1}$ and can be evaluated numerically from (6). Obviously, for large number of sub-carriers, $N$, this might be a complex optimization problem.

Applying a sub-optimal uniform power allocation, $p_{n}=$ $P \forall n$, to (6) yields

$$
R_{1 \text { bit-upa }}(\boldsymbol{\alpha})=\frac{1}{N} \sum_{n=0}^{N-1} Q\left(\alpha_{n} / \sigma_{n}^{2}, K\right) \mathcal{F}\left(\alpha_{n} / \sigma_{n}^{2}, \sigma_{n}^{2} P\right) \text {. }
$$

Examining (8), reveals that the optimal thresholds for the uniform power allocation can be calculated numerically for each sub-carrier contributing term.

\section{Sub-Optimal Thresholds AND Asymptotic ANALYSIS}

To lower bound the achievable sum-rate (6) we note that $\mathcal{F}(x, y) \geq \log (1+x y), \forall x, y \geq 0$. Hence, the sum-rate is lower bounded by

$$
R_{\mathrm{lb}}(\boldsymbol{\alpha})=\max _{\boldsymbol{p}:|\boldsymbol{p}| \leq N P} \frac{1}{N} \sum_{n=0}^{N-1} Q\left(\alpha_{n} / \sigma_{n}^{2}, K\right) \log \left(1+p_{n} \alpha_{n}\right) .
$$

Comparing the lower bound (9), to the asymptotic capacity of the full-CSI scheme (2), it is clear that a "good" threshold vector is such that for $K \gg 1$ the pre-log terms $\left\{Q\left(\alpha_{n} / \sigma_{n}^{2}, K\right)\right\}_{n=0}^{N-1}$ approach 1 and the multiuser diversity gains are close to $\sigma_{n}^{2} \log K$. By setting the following suboptimal thresholds [10]

$$
\alpha_{n}^{\text {so }}=\sigma_{n}^{2}(\log K-\log \log K),
$$

these two requirements are fulfilled since,

$$
Q\left(\alpha_{n}^{\mathrm{so}} / \sigma_{n}^{2}, K\right)=q(K) \triangleq\left(1-\left(1-\frac{\log K}{K}\right)^{K}\right) \underset{K \rightarrow \infty}{\rightarrow} 1
$$

and

$$
\frac{\alpha_{n}^{\text {so }}}{\sigma_{n}^{2} \log K}=\frac{\sigma_{n}^{2}(\log K-\log \log K)}{\sigma_{n}^{2} \log K} \underset{K \rightarrow \infty}{\rightarrow} 1 .
$$

Substituting the sub-optimal thresholds (10) into (9), the lower bound boils down to

$R_{\mathrm{lb}}\left(\boldsymbol{\alpha}^{\mathrm{so}}\right)=\frac{q(K)}{N} \max _{\boldsymbol{p}:|\boldsymbol{p}| \leq N P} \sum_{n=0}^{N-1} \log \left(1+p_{n} \sigma_{n}^{2} \log \frac{K}{\log K}\right)$.

The waterfilling solution to the standard optimization problems of (13) is given by

$$
p_{n}=\left(\frac{1}{\lambda}-\frac{1}{\sigma_{n}^{2} \log \frac{K}{\log K}}\right)^{+} \text {s.t. }|\boldsymbol{p}|=N P,
$$

and for uniform power allocation the lower bound is given by

$$
R_{\mathrm{lb}-\mathrm{upa}}\left(\boldsymbol{\alpha}^{\mathrm{so}}\right)=\frac{q(K)}{N} \sum_{n=0}^{N-1} \log \left(1+P \sigma_{n}^{2} \log \frac{K}{\log K}\right) \text {. }
$$

Adhering to similar argumentation regarding the channel statistics as made in Section IV, we claim that for a finite $P$ and large number of users $K$, the waterfilling solution of (14) converges to the uniform power allocation solution of (15). Now, since

$$
\frac{R_{\mathrm{lb}-\mathrm{upa}}\left(\boldsymbol{\alpha}^{\mathrm{so}}\right)}{\log \log K} \underset{K \rightarrow \infty}{\rightarrow} 1,
$$

and by noting that the ergodic sum-rate for an optimal thresholds selection, $\boldsymbol{\alpha}^{\mathrm{o}}$, is lower bounded by (15) and is upper bounded by the full-CSI ergodic sum-rate capacity

$$
R_{\mathrm{lb}-\mathrm{upa}}\left(\boldsymbol{\alpha}^{\mathrm{so}}\right) \leq R_{1 \mathrm{bit}}\left(\boldsymbol{\alpha}^{\mathrm{o}}\right) \leq C_{\mathrm{fcsi}},
$$


it is concluded that the ergodic sum-rate of the 1-bit feedback scheme grows as $\log \log K$ asymptotically with the number of users which is the same growth rate achieved by the full-CSI scheme.

Next, we consider the high-SNR ergodic sum-rate slope of the 1-bit feedback scheme which satisfies

$$
\begin{aligned}
S_{\infty}^{1 \mathrm{bit}} & =\lim _{P \rightarrow \infty} \frac{R_{1 \mathrm{bit}}\left(\boldsymbol{\alpha}^{\mathrm{o}}\right)}{\log P} \geq \lim _{P \rightarrow \infty} \frac{R_{\mathrm{lb}}\left(\boldsymbol{\alpha}^{\mathrm{so}}\right)}{\log P} \\
& =\lim _{P \rightarrow \infty} \frac{R_{\mathrm{lb}-\text { upa }}\left(\boldsymbol{\alpha}^{\mathrm{so}}\right)}{\log P}=q(K)_{K \rightarrow \infty}^{\rightarrow} 1 .
\end{aligned}
$$

where the second equality is justified by adhering to similar argumentation as made for the full-CSI scheme regarding the channel statistics, and claiming that the waterfilling solution of (14) has little effect at the high-SNR regime. Hence, the uniform power allocation solution of (15) is optimal in the high-SNR regime. The third equality is derived by applying the limit to (15). Finally, by recalling (11) and (17), it is concluded that the high-SNR ergodic sum-rate slope of the 1-bit feedback scheme approaches the high-SNR sum-rate capacity slope of the full-CSI scheme (5) asymptotically with the number of users.

\section{NUMERICAL RESULTS}

In figure 1, curves of the average spectral efficiencies per sub-carrier ${ }^{3}$ of the analyzed setups as a function of the average transmitted $E_{b} / N_{0}$, for $K=100$ and $N=256$, are shown. In addition, a truncated and shifted raised cosine pulse with span of 9 [samples] and rolloff factor of $\beta=0.3$ is used to shape the transmitted signals. The tested channel is composed of $N_{p}=3$ paths with average powers $\{0.6,0.3,0.1\}$ and paths delays of $\left\{0.5 T_{\mathrm{s}}, 16.5 T_{\mathrm{s}}, 40.5 T_{\mathrm{s}}\right\}$ ( $T_{\mathrm{s}}$ is the sample duration). The overall paths' power gains is normalized to 1 in order to provide a proper comparison to the reference AWGN channel. This channel provides a worst-case scenario (for a given rolloff factor), since its average gains decrease to zero for sub-carriers indices above the cutoff frequency (for rolloff factor of $\beta=0.3$ and $N=256$ sub-carriers the cutoff index is around the 90th sub-carrier).

Figure 1 includes the following spectral efficiencies curves: (a) full-CSI scheme with optimal power allocation (expression (2)), (b) full-CSI scheme with uniform power allocation (expression (4)), (c) 1-bit feedback scheme with uniform power allocation and optimal thresholds (expression (8)), and (d) reference AWGN channel. It is observed that the 1-bit feedback scheme demonstrates a small loss (less than $0.5[\mathrm{bit} / \mathrm{sec} / \mathrm{Hz}]$ ), due to the limited 1-bit feedback, when compared to the sum-rate capacity of full-CSI scheme. Note that the actual loss may be smaller since the spectral efficiency of the 1-bit feedback scheme with uniform power and optimal thresholds is obviously a lower bound to the ultimate sum-rate achieved

\footnotetext{
${ }^{3}$ The average spectral efficiency per sub-carrier in [bits/sec/Hz], expressed as a function of the system average transmit $E_{b} / N_{0}, E_{b}^{t} / N_{0}$, is evaluated by solving the implicit equation obtained by substituting $P=\mathrm{C}\left(\frac{E_{b}^{t}}{N_{0}}\right) \frac{E_{b}^{t}}{N_{0}}$, where $\mathrm{C}\left(E_{b}^{t} / N_{0}\right)=C(P)$ stands for the average spectral efficiency per sub-carrier of the different setups.
}

by the 1-bit feedback scheme. It is also observed that the optimal power allocation by waterfilling is beneficial only in the low-SNR regime. Finally, both schemes with full-CSI and 1-bit feedback, demonstrate a multiuser diversity gain when compared to the spectral efficiency of the reference AWGN channel.

\section{Nonhomogeneous Channel Model}

In previous sections a homogenous model was considered (see Appendix A). According to this model, all users experience identical fading paths delays. Moreover, the average powers of the paths gains are also identical for all users. In this section, the strict assumptions are relaxed, and both paths delays and gains are no longer assumed to be equal for all users. However, the total average power of the paths gains remains equal for all users, i.e.,

$$
\sigma_{a}^{2} \triangleq \sum_{l=1}^{N_{\mathrm{p}}(k)} \sigma_{a}^{2}(l, k) \quad ; \quad \forall k,
$$

where $N_{\mathrm{p}}(k)$ is the number of fading paths experienced by the $k$ th user and $\left\{\sigma_{a}^{2}(l, k)\right\}_{l=1}^{N_{\mathrm{p}}(k)}$ are its corresponding gains average powers. It is noted that although the paths' delays are no longer identical, it is expected that they do not vary significantly since it is assumed that the main scatterers are located near the transmitting antenna.

Since in real life scenarios different systems share adjacent frequency bands, emerging OFDM based systems such as IEEE 802.16 based systems, do not allocate power to near Nyquist sub-carriers, in order to reduce the mutual interference. One of the conclusions of Appendix A, is that regardless of the actual path delay, and assuming that the shaping pulse span is properly chosen, each path provides a "flat" contribution to the overall sub-carrier average power gain vector over the pass-band frequencies. Hence, by utilizing sub-carriers which lie in the pass-band only, the effective subcarriers average gain powers are practically constant and equal

$$
\sigma_{n}^{2}=\sigma_{a}^{2} \quad ; \quad n=0,1, \ldots, N_{\text {co }},
$$

where $\sigma_{a}^{2}$ is defined in (19), and the index of the highest active sub-carrier, $N_{\text {co }}$, can be adjusted by a proper selection of the rolloff factor of the raised-cosine shaping pulse.

Accounting for the above assumptions and restrictions, it is obvious that uniform power allocation (over the active subcarriers) $p_{n}=P \forall n \in\left[0, N_{\mathrm{co}}\right]$ and uniform thresholds $\alpha_{n}=\alpha \forall n \in\left[0, N_{\mathrm{co}}\right]$, are optimal. Respectively, the ergodic achievable sum-rate per active sub-carrier of the 1-bit feedback scheme, is derived by substituting (20) into (8), and is given by

$$
R_{1 \mathrm{bit}-\mathrm{nh}}(\alpha) \cong Q\left(\alpha / \sigma_{a}^{2}, K\right) \mathcal{F}\left(\alpha / \sigma_{a}^{2}, \sigma_{a}^{2} P\right),
$$

where $Q(\cdot)$ and $\mathcal{F}(\cdot)$ are defined by (7). It is noted that (21) is the same as the rate of the single-carrier setup analyzed in [11]. In addition, it is easily verified that (21) shares the same asymptotic behavior of the rate achieved for the homogenous model (see Section VI). 


\section{Conclusions}

In this paper we analyze the ergodic sum-rate of the 1-bit feedback per fading block per sub-carrier scheme presented in [1] for a SISO downlink channel. It is concluded that the scheme which significantly reduces the feedback needed when compared to the feedback needed for the full-CSI scheme, achieves asymptotically with the number of users, optimal sum-rate growth and non-interference limited behavior. Achieving the same high-SNR sum-rate capacity slope as the one achieved by the optimal scheme, demonstrates the special case encompassed by the SISO setup, in which the highSNR sum-rate capacity slope (or degrees of freedom) may not decrease when partial CSI is available to the transmitter. This fact comes in contrast to the behavior of a MIMO setup, where it has been recently shown that partial CSI reduces the highSNR sum-rate capacity slope [12]. Finally, numerical results show that for an appropriate thresholds selection, and even for a moderate number of users, the scheme demonstrates a spectral efficiency loss of fraction of $[\mathrm{bit} / \mathrm{sec} / \mathrm{Hz}]$ when compared to the fully informed scheme, and that optimal power allocation provides little benefit (only at low-SNR values) for the channel model considered.

\section{APPENDIX}

\section{A. Channel Statistics}

The equivalent channel impulse response (assuming perfect symbol and block synchronization between the transmitter and receivers) is given for an arbitrary user and fading block by

$$
h_{m}=\sum_{l=1}^{N_{\mathrm{p}}} a_{l} s\left(m T_{\mathrm{s}}-\tau_{l}\right),
$$

where $T_{\mathrm{s}}$ is the sample duration, $N_{\mathrm{p}}$ is the number of independent fading paths, $\left\{\tau_{l}\right\}_{l=1}^{N_{\mathrm{p}}}$ are the respective paths delays (much smaller than the OFDM symbol duration), and $\left\{a_{l}\right\}_{l=1}^{N_{\mathrm{p}}}$ are the statistically independent paths gains $a_{l} \sim$ $\mathcal{C N}\left(0, \sigma_{a}^{2}(l)\right)$. In addition,

$$
s(t)=\left\{\begin{array}{ll}
s_{\mathrm{rc}}^{\beta}\left(t-L_{\mathrm{s}} T_{\mathrm{s}}\right), & 0 \leq t \leq 2 L_{\mathrm{s}} T_{\mathrm{s}} \\
0, & \text { otherwise }
\end{array},\right.
$$

where $s_{\mathrm{rc}}^{\beta}(t)$ is a raised-cosine function with a rolloff factor $\beta$ and a bandwidth of $1 / T_{\mathrm{s}}$ [13]. Hence, $s(t)$ is a truncated and shifted raised-cosine function, which spans over $2 L_{\mathrm{s}}+1$ samples $\left(L_{\mathrm{s}} \ll N\right)$. It is noted, that the delay spread of the equivalent channel is $\tilde{T}_{\mathrm{ds}} \leq T_{\mathrm{ds}}+2\left(L_{\mathrm{s}}+1\right) T_{\mathrm{s}}$ (where $T_{\mathrm{ds}}$ is the original delay spread of the channel) and the cyclic prefix should be set accordingly. It is noted that signal shaping is used by OFDM system in order to reduce the transmission side-lobes and to mitigate the interference to other systems operating over adjacent frequency bands. At the receivers end, the same pulse is used for match-filtering the incoming signal. Accordingly, in the current setup, a truncated and shifted square-root raised-cosine function is used, resulting in the equivalent truncated and shifted raised-cosine function of (23).
It is easily verified that the coefficients of the equivalent channel impulse response are zero mean complex Gaussian r.v.'s, and their covariance coefficients are given by

$$
r_{q_{1}, q_{2}}=E\left\{h_{q_{1}} h_{q_{2}}^{\dagger}\right\}=\sum_{l=1}^{N_{p}} \sigma_{a}^{2}(l) s\left(q_{1} T_{\mathrm{s}}-\tau_{l}\right) s\left(q_{2} T_{\mathrm{s}}-\tau_{l}\right) .
$$

Now, the ground is set to calculate the $n$th sub-carrier gain average power for an arbitrary user, which is given by

$$
\begin{aligned}
\sigma_{n}^{2} & =E\left\{\left|\sum_{k=0}^{N-1} h_{k} e^{-\frac{j 2 \pi k n}{N}}\right|^{2}\right\}=\sum_{q_{1}=0}^{N-1} \sum_{q_{2}=0}^{N-1} r_{q_{1}, q_{2}} e^{\frac{j 2 \pi\left(q_{2}-q_{1}\right) n}{N}} \\
& =\sum_{l=1}^{N_{p}} \sigma_{a}^{2}(l) \sum_{q_{1}=0}^{N-1} \sum_{q_{2}=0}^{N-1} s\left(q_{1} T_{\mathrm{s}}-\tau_{l}\right) s\left(q_{2} T_{\mathrm{s}}-\tau_{l}\right) e^{\frac{j 2 \pi\left(q_{2}-q_{1}\right) n}{N}} \\
& =\sum_{l=1}^{N_{p}} \sigma_{a}^{2}(l)\left|S_{n}\left(\tau_{l}\right)\right|^{2}
\end{aligned}
$$

where the third equality is achieved by substituting (24), and changing the summation order. In addition, $\left\{S_{n}\left(\tau_{l}\right)\right\}$ is the DFT of the sampled shaping signal with offset $\tau_{l}$,

$S_{n}\left(\tau_{l}\right) \triangleq \sum_{k=0}^{N-1} s\left(k T_{\mathrm{s}}-\tau_{l}\right) e^{-\frac{j 2 \pi k n}{N}} \quad ; \quad n=0,1, \ldots N-1$.

It is noted that $\left\{\left|S_{n}\left(\tau_{l}\right)\right|^{2}\right\}$ is actually a function of the difference between the path delay and the nearest left-most sample $\tilde{\tau}_{l}=\tau_{l}-\tau_{l} \bmod T_{\mathrm{s}}$, and not of the actual value of $\tau_{l}$.

In Figure 2, $\left\{\left|S_{n}\left(\tilde{\tau}_{l}\right)\right|^{2}\right\}$ is plotted for $N=256$, raised cosine rolloff factor of $\beta=0.3$, raised-cosine span of $2\left(L_{\mathrm{s}}+1\right)=9 T_{\mathrm{s}}$, and several values of $\tilde{\tau}_{l}$. It is observed that the case where $\tilde{\tau}_{l}=0.5 T_{\mathrm{s}}$ provides a worst-case scenario in terms of the sub-channels gains, while the case where $\tilde{\tau}_{l}=0$ (meaning that the path delay coincides with the sampling process), provides a flat contribution to the overall sub-carriers average gain vector. The latter is easily verified from (25) and the fact that the raised cosine function satisfies the Nyquist pulse shaping criterion. Another observations is a cutoff phenomenon for $\tilde{\tau}_{l}>0$, resulting from the fact that $\left\{\left|S_{n}\left(\tilde{\tau}_{l}\right)\right|^{2}\right\}$ is closely related to the Fourier transform of the raised-cosine function. Accordingly, the cutoff frequency decreases with increasing values of the raised-cosine rolloff factor $\beta$. In addition, a windowing effect is observed across the pass-band frequencies, where the ripple of the sub-channels gains increases for decreasing span values [8]. It is noted that for a proper selection of the raised-cosine span, $\left\{\left|S_{n}\left(\tilde{\tau}_{l}\right)\right|^{2}\right\}$ practically provides a "flat" contribution to the over all subchannels gains within the pass-band frequencies, regardless of the actual value of $\tilde{\tau}_{l}$.

\section{B. Ergodic Sum-Rate Derivation}

According to the 1-bit feedback scheme described in Section III, the power allocation and threshold vectors depend on the long term statistics of the channel (and not on the instantaneous CSI), and are predetermined. In addition, at 
any time instance, no more than one user is active in each sub-carrier. Assuming an independent single user encodingdecoding scheme for each user on each sub-carrier over many OFDM symbols (and many fading blocks), the ergodic sumrate achieved when the $n$th sub-carrier is active is given by

$$
\tilde{R}_{n}\left(\alpha_{n}, p_{n}\right)=E_{\left|\tilde{H}_{n}\right|^{2}}\left\{\log \left(1+p_{n}\left|\tilde{H}_{n}\right|^{2}\right)\right\},
$$

where $\left|\tilde{H}_{n}\right|^{2}$ is an arbitrary fade power level which is conditionally distributed above a certain level $\alpha_{n}$, and $p_{n}$ is the allocated power. It is easy to verify that the probability distribution function of $\left|\tilde{H}_{n}\right|^{2}$ is given by

$$
f_{\left|\tilde{H}_{n}\right|^{2}}(x)=\frac{1}{\sigma_{n}^{2}} \exp \left(\frac{\alpha_{n}-x}{\sigma_{n}^{2}}\right) \quad ; \quad x \in\left[\alpha_{n}, \infty\right),
$$

with mean value $E\left\{\left|\tilde{H}_{n}\right|^{2}\right\}=\alpha_{n}+\sigma_{n}^{2}$. Applying the integration by part formula to (27) it is easily verified that

$$
\tilde{R}_{n}\left(\alpha_{n}, p_{n}\right)=\mathcal{F}\left(\alpha_{n} / \sigma_{n}^{2}, \sigma_{n}^{2} p_{n}\right),
$$

where $\mathcal{F}(\cdot)$ is defined by (7). Since, the $n$th sub-carrier is active only if there is at least one user with fade power level above the threshold, the ergodic sum-rate of the $n$th sub-carrier is given by multiplying (29) by the average fraction of time in which the sub-carrier is active

$$
\begin{aligned}
P_{r}\left(N_{n}>0\right) & =1-P_{r}\left(N_{n}=0\right)=1-\left(P_{r}\left(\left|H_{n}\right|^{2}<\alpha_{n}\right)\right)^{K} \\
& =1-\left(1-\exp \left(-\frac{\alpha_{n}}{\sigma_{n}^{2}}\right)\right)^{K} .
\end{aligned}
$$

where $N_{n}$ is the number of users with fade powers above the threshold $\alpha_{n}$, and $\left|H_{n}\right|^{2}$ is an arbitrary chi-square random variable with two degrees of freedom and expected value of $\sigma_{n}^{2}$. Hence, the ergodic sum-rate of the $n$th channel is given by

$$
\begin{aligned}
R_{n}\left(\alpha_{n}, p_{n}\right) & =P_{r}\left(N_{n}>0\right) \tilde{R}_{n}\left(\alpha_{n}, p_{n}\right) \\
& =Q\left(\alpha_{n} / \sigma_{n}^{2}, K\right) \mathcal{F}\left(\alpha_{n} / \sigma_{n}^{2}, \sigma_{n}^{2} p_{n}\right),
\end{aligned}
$$

where $Q(\cdot)$ is defined by (7). Finally, summing over all the rates of all sub-carriers and maximizing the total sum-rate by allocating the constrained power $N P$ to the sub-carriers, complete the derivation.

\section{REFERENCES}

[1] S. Sanayei, A. Nosratinia, and N. Aldhahir, "Opportunistic dynamic subchannel allocation in multiuser OFDM networks with limited feedback," in Proceedings of the IEEE Information Theory Workshop (ITW04), (San Antonio, Texas, USA), Oct. 24-29 2004.

[2] D. Tse, "Optimal power allocation over parallel broadcast Gaussian channels," in Proc. International Symposium on Information Theory (ISIT'97), (Ulm, Germany), Jun. 1997.

[3] D. Gesbert and S. Alouini, "Selective multiuser diversity," in Proc. of Intl. Symp. on Sig. Proc. and Info. Techn. (ISSPIT'03), (Darmstadt, Germany), Dec. 2003.

[4] D. Gesbert and S. Alouini, "How much feedback is multi-user diversity really worth," in Proc. International Conference on Communications (ICC'04), (Paris, France), Jun. 2004.

[5] P. Svedman, S. K. Wilson, L. Cimini, and B. Ottersten, "A simplified feedback and scheduling scheme for OFDM," in Proceedings of Vehicular Technology Conference, (Milan, Italy), May. 17-19 2004.

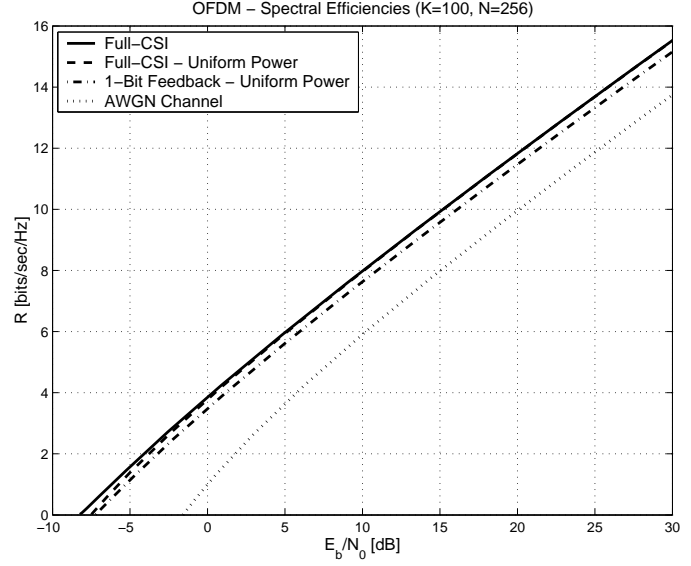

Fig. 1. Spectral efficiencies for $K=100$ users and $N=256$ sub-carriers.

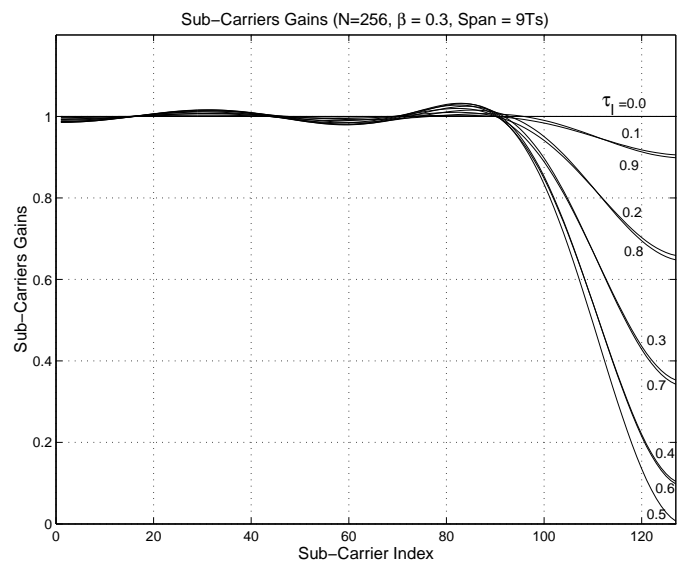

Fig. 2. Sub-carriers gains for a truncated and shifted raised-cosine pulse with $\beta=0.3$ and $L_{\mathrm{s}}=9 T_{\mathrm{s}}$, for $N=256$ and several values of $\tilde{\tau}_{l}$

[6] S. Sanayei and A. Nosratinia, "Exploiting multiuser diversity with only 1-bit feedback," in Proceedings of the IEEE Wireless Communications and Networking Conference (WCNC'05), (New Orleans, LA, USA), Mar. 2005.

[7] M. Sharif and B. Hassibi, "On the capacity of MIMO broadcast channel with partial side information," IEEE Transactions on Information Theory, vol. 51, pp. 506-522, Feb. 2005.

[8] O. Somekh, O. Simeone, A. M. Haimovich, and Y. Bar-Ness, "Sum-rate analysis of general OFDM downlink channels with 1-bit feedback per sub-carrier." In Preparation.

[9] A. Lozano, A. M. Tulino, and S. Verdú, "High-SNR power offset in multimntenna communication," IEEE Transactions on Information Theory, vol. 51, pp. 4134-4151, Dec. 2005.

[10] J. Diaz, O. Simeone, O. Somekh, and Y. Bar-Ness, "Scaling law of the sum-rate for multiantenna broadcast channels with deterministic or selective binary feedback," in Proceedings of the IEEE Information Theory Workshop (ITW'06), (Punta Del Esta, Uruguay), Mar. 13-17 2006.

[11] O. Somekh, A. M. Haimovich, and Y. Bar-Ness, "Sum-rate analysis of dowlink channels with 1-bit feedback." Submitted to the IEEE Communication Letters.

[12] A. Lapidoth, S. Shamai (Shitz), and M. A. Wigger, "On the capacity of fading mimo broadcast channels with imperfect transmitter sideinformation," in Proceedings of 43rd Annual Allerton Conference on Communication, Control, and Computing, (Allerton), Sep. 28-30 2005.

[13] J. G. Proakis, Digital Communications. New York: McGraw-Hill, third ed., 1995. 